\title{
A Relay Controller with Parallel Feed-forward Compensation for a Buck Converter Feeding Constant Power Loads
}

\author{
Roya Rafiezadeh* and Robert Griñó* \\ *Institute of Industrial and Control Engineering (IOC) \\ Universitat Politècnica de Catalunya (UPC) - Barcelona Tech \\ Barcelona, Spain \\ \{roya.rafiezadeh, roberto.grino\}@upc.edu
}

\begin{abstract}
A simple and efficient analog direct voltage control design for improving the performance and stability of a buck converter feeding constant power loads (CPLs) is the main aim of this article. One of the oldest and most spread approaches is to design a relay control system. In these systems, although the hysteretic relay adds non-linearity to the closed-loop system, an adequate controller design procedure will be presented using the locus of a perturbed relay system (LPRS) technique. This work shows how a designer can apply LPRS technique to a converter plant, in this case a buck converter feeding a mixed linear and constant power load (CPL), using a parallel feedforward compensator (PFC) to obtain a suitable performance in the closed-loop system. That means to enlarge the range of stable behavior with respect to the power in the CPLs, in comparison with the open-loop case, and to regulate adequately the output voltage of the converter in front of input voltage and load power variations. The simulation results with the converterload non-linear model show the good performance of the designed controller with respect to the above-mentioned disturbances and perturbations.
\end{abstract}

Index Terms - constant power load, relay feedback system, almost strictly positive real, parallel feed-forward compensator, locus of a perturbed relay system.

\section{INTRODUCTION}

Nowadays, the use of power converters in electrical systems is increasing due to having the ability and characteristic to accumulate and transform electrical energy. The performance of power electronic converters depends on the variations of the output (voltage and current) due to fluctuations of the source voltage, internal parameters changes, and external disturbances. In addition, recently, most of these power converters feed mixed loads which are linear loads in parallel with constant power loads (CPLs). Owing to the non-linear behavior of CPLs, that threaten the stability of electrical systems, the performance of power converters will be compromised in comparison with feeding linear positive resistance loads. Different approaches for improving the stability of the electrical circuits consisting CPLs have been proposed in [1]-[4]. So, because of these reasons the development of a practical simple control strategy, convenient for the stability and performance of power converters, and also to be able to cope more with the plant uncertainties and disturbances is always required. From control perspective, depending on the designers parameters assumptions and plant characteristics, the desired control strategy will be defined. Various control strategies (analog or digital) are applied to power converters for improving their stability and efficiency, for example a good comparison of them can be found in [5]. In this work, we are more interested on analog controllers due to being more simple and cheaper than the other options which are implemented in micro-controllers and DSPs, and also on direct voltage control techniques nor the typical cascade structure of control loops. Among the oldest, powerful and widespread control techniques, the advantages of using relay feedback systems (RFS) in general and hysteretic controlled systems in particular, which are also extended in control of power electronic converters [6] and [7], have been proved in the form of their wide presence in electronic applications. Moreover, the use of RFS with feedback loops presents simplicity, cheapness, great performance, and wide robustness margins. This paper proposes RFS controller for improving the performance and stability of a dc/dc buck converter feeding a mixed load while handling more output power in a stable manner. This approach is based on using a parallel feed-forward compensator (PFC) [8]-[12], locally feeding back the relay controller to ensure the stability of the closed-loop while sweeping the power of the CPL. The PFC is also rely on the concept of almost strictly positive real (ASPR) function [13], and non-minimum phase plants. In addition, locus of a perturbed relay system (LPRS) [14], is used as a technique to determine the exact frequency and amplitude of the self-excited oscillations (chattering), and also the value of the RFS.

Section 2 describes the analysis of a dc/dc buck converter feeding a mixed load. Section 3 discusses the architecture of the control system. Section 4 and Section 5 present the controller design and simulation results, respectively. Finally, Section 6 reports the main conclusion of this work. 


\section{ANAlysis OF THE DC/DC BUCK CONVERTER}

Conventional $\mathrm{dc} / \mathrm{dc}$ buck converter with resistive load, in average, is a linear electrical circuit; while some special loads can add non-linearity to it. One of these loads is constant power load, CPL, that adds non-linearity to the converter behavior. The CPL-buck converter may produce a constant power for different loads; or equivalent, but different loads with the same output voltage get different constant currents from the converter. In fact, it may consider as a negative non-linear resistance and it can be modeled as a dependent current source. Fig. 1 shows an averaged model of a dc/dc buck converter feeding mixed load, consisting input voltage source $(E)$, control signal $(u)$ while $u \in\{0,1\}$, parasitic resistance of inductor $(r)$, inductor $(L)$, output capacitor $\left(C_{o}\right)$, load resistor $(R)$, inductor current $\left(i_{L}\right)$, capacitor current $\left(i_{C}\right)$, output current $\left(i_{o}\right)$, load resistance current $\left(i_{R}\right)$, and CPL current $\left(i_{C P L}=\frac{P_{o}}{v_{o}}\right)$.

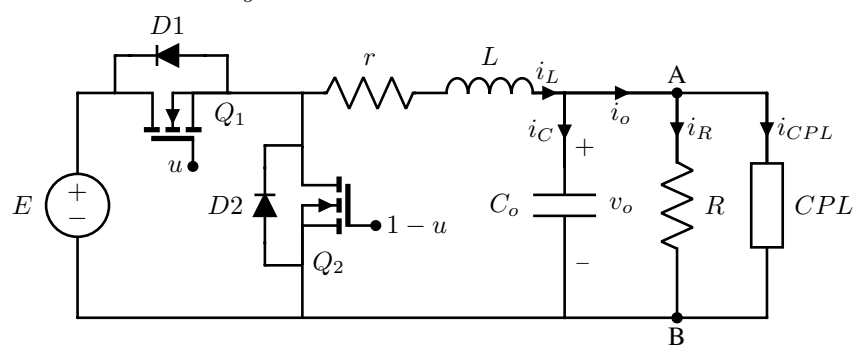

Fig. 1: A current bi-directional buck converter feeding mixed load.

By Thévenin theorem, the buck converter from the points $\mathrm{AB}$ can be replaced by an equivalent voltage source $\left(E_{t h}\right)$ series with an impedance $\left(Z_{t h}\right)$, which will be obtained as

$$
\begin{aligned}
& E_{t h}(s)=F(s) u, \\
& Z_{t h}(s)=\frac{\frac{1}{C_{o}} s+\frac{r}{L C_{o}}}{s^{2}+\frac{r}{L} s+\frac{1}{L C_{o}}},
\end{aligned}
$$

where $F(s)$ is the transfer function of the circuit, seen from points $\mathrm{AB}$ and is equal to

$$
F(s)=\frac{\frac{E}{L C_{o}}}{s^{2}+\frac{r}{L} s+\frac{1}{L C_{o}}} .
$$

In Fig. 2, the Thévenin equivalent circuit of a buck converter feeding mixed load seen at points $A B$ is shown. In this figure, the non-linear component is modeled as a dependent current source $\left(i_{C P L}\right)$ and the output voltage is

$$
V_{o}(s)=-Z_{t h}(s) I_{o}(s)+E_{t h}(s) .
$$

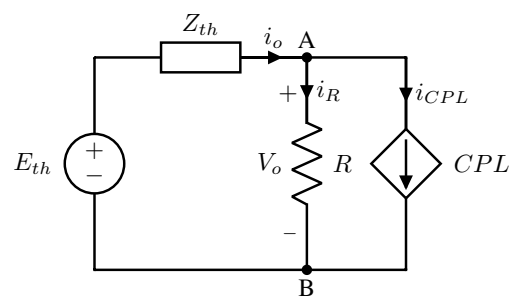

Fig. 2: Thévenin equivalent of a buck converter feeding mixed load.
By Taylor series expansion, a linear representation of the dependent current, $i_{C P L}=\frac{P_{o}}{v_{o}}$, is

$$
\begin{aligned}
& \Delta i_{C P L}=\left.\left(\partial i_{C P L} / \partial v_{o}\right)\right|_{\left(\bar{P}_{o}, \bar{v}_{o}\right)} \Delta v_{o}+ \\
& \left.\left(\partial i_{C P L} / \partial P_{o}\right)\right|_{\left(\bar{P}_{o}, \bar{v}_{o}\right)} \Delta P_{o}=\left(-\bar{P}_{o} / \bar{v}_{o}^{2}\right) \Delta v_{o}+\left(1 / \bar{v}_{o}\right) \Delta P_{o},
\end{aligned}
$$

where $\bar{P}_{o}$ and $\bar{v}_{o}$ are the values of the CPL power and output voltage at the equilibrium, respectively; moreover, as $P_{o}$ is constant then, $\Delta P_{o}=0$. With (4) the block diagram of the linear buck converter is shown in Fig. 3, in which $G_{R}=1 / R$, is the conductance of the linear load and $G_{C P L}(s)=-\bar{P}_{o} / \bar{v}_{o}^{2}$ is the incremental conductance of the CPL.

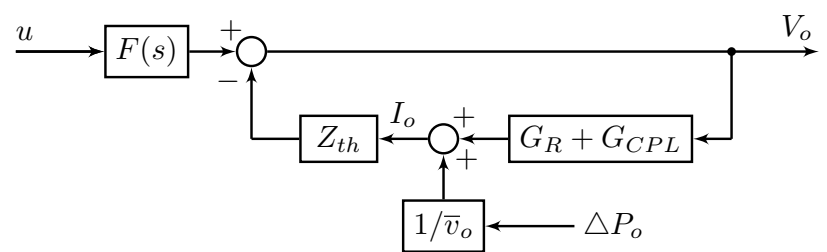

Fig. 3: Block diagram of the linear mixed load buck converter.

Considering Fig. 3, and $G_{l}=G_{R}+G_{C P L}$, (3) can be modified as

$$
V_{o}(s)=\frac{F(s)}{1+Z_{t h}(s) G_{l}(s)} U(s)-\frac{\left(1 / \bar{v}_{o}\right) Z_{t h}(s)}{1+Z_{t h}(s) G_{l}(s)} \Delta P_{o}(s) \text {. }
$$

The state-space equations of the non-linear CPL-buck converter parallel with a conductivity where the output capacitor voltage is the first state and the inductor current is the second state, $x=\left[v_{o}, i_{L}\right]^{T}$, are

$$
\begin{aligned}
& C_{o} \dot{x}_{1}=-G_{R} x_{1}-\frac{P_{o}}{x_{1}}+x_{2}, \\
& L \dot{x}_{2}=-x_{1}-r x_{2}+u E .
\end{aligned}
$$

In the following, the linearization procedure of the nonlinear CPL-buck converter is explained. In this procedure, at first, the equilibrium points of the state-space equations in (6) are found, then by Taylor's series, the linear state-space equations of the system are extracted.

\section{A. Equilibrium Points}

With respect to (6), by $\dot{x}_{1}=0, \dot{x}_{2}=0$, and constant $v_{o}=$ $\bar{v}_{o}$, the result of equilibrium points for the mixed load buck converter are

$$
\begin{aligned}
& \bar{i}_{L}=G_{R} \bar{v}_{o}+\frac{P_{o}}{\bar{v}_{o}}, \\
& \bar{u}=\frac{\left(1+G_{R} r\right) \bar{v}_{o}^{2}+r P_{o}}{\bar{v}_{o} E},
\end{aligned}
$$

where $0 \leq \bar{u} \leq 1$. By solving inequality $\bar{u} \leq 1$ for $P_{o}$, the upper limit of output power is

$$
P_{o} \leq P_{o_{\max }}=\frac{\left(E-\left(1+G_{R} r\right) \bar{v}_{o}\right) \bar{v}_{o}}{r},
$$

which shows the range of CPL power for existence of equilibrium. 


\section{B. Plant Linearization}

In general, Taylor series is a useful technique for linearizing a non-linear plant. Considering the minimal state-space system

$$
\begin{aligned}
& \dot{x}=A x+B_{1} u+B_{2} \Delta P_{o}, \\
& y=C x+D u,
\end{aligned}
$$

where $A \in \mathbb{R}^{2 \times 2}, B_{1} \in \mathbb{R}^{2 \times 1}, B_{2} \in \mathbb{R}^{2 \times 1}, C \in \mathbb{R}^{1 \times 2}$, and $D \in \mathbb{R}^{1 \times 1}$. The non-linear part of (6) is $P_{o} / x_{1}$ and by Taylor series, its linear form is $-\left(\bar{P}_{o} / \bar{v}_{o}^{2}\right) x_{1}+\left(1 / \bar{v}_{o}\right) \Delta P_{o}$; accordingly, the linear state-space matrices of (6) are

$$
\begin{gathered}
A=\left[\begin{array}{cc}
\frac{\bar{P}_{o}}{C_{o} \bar{v}_{o}^{2}}-\frac{G_{R}}{C_{o}} & \frac{1}{C_{o}} \\
-\frac{1}{L} & -\frac{r}{L}
\end{array}\right], B_{1}=\left[\begin{array}{c}
0 \\
\frac{E}{L}
\end{array}\right], B_{2}=\left[\begin{array}{c}
-\frac{1}{C_{o} \bar{v}_{o}} \\
0
\end{array}\right], \\
C=\left[\begin{array}{ll}
1 & 0
\end{array}\right], D=0 .
\end{gathered}
$$

Considering linear state-space matrices A, B, C, and D, the transfer function of the buck converter feeding mixed load, in Fig. 1 will be ${ }^{1}$

$$
G_{u}(s)=\frac{Y(s)}{U(s)}=\frac{b_{0}}{s^{2}+a_{1} s+a_{0}},
$$

where

$b_{0}=\frac{E}{L C_{o}}, a_{1}=\frac{r}{L}+\frac{G_{R}}{C_{o}}-\frac{\bar{P}_{o}}{\bar{v}_{o}^{2} C_{o}}, a_{0}=\frac{r G_{R}}{L C_{o}}+\frac{1}{L C_{o}}-\frac{r \bar{P}_{o}}{\bar{v}_{o}^{2} L C_{o}}$.

Obviously, the CPL power, $P_{o}$, and the load conductance, $G_{R}$, affect the plant stability. Applying the Routh's criterion, the plant will be stable if and only if inequalities $a_{0}>0$ and $a_{1}>0$ are met. From them, the maximum value of the CPL power to have a stable plant, $P_{o}^{*}$, is

$$
P_{o}^{*}<\frac{r C_{o}+G_{R} L}{L} \bar{v}_{o}^{2}
$$

\section{ARChitecture of the CONTROL System}

This section presents the main considerations and the architecture of the control system. In this work, a direct output voltage control of a buck converter feeding mixed load with no measuring the inductor current is proposed. Furthermore, the controller should have a binary output signal that will be directly applied to the switches as is shown in Fig. 4 where $C(s)$ is the controller, $v_{o_{r e f}}$ and $e$ are the output voltage reference and the error signals, respectively.

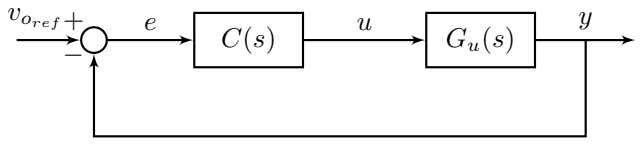

Fig. 4: Closed-loop control block diagram while $u$ is control signal.

For designing the controller, we will mainly use relay feedback systems (RFS) which are non-linear systems [15], and this non-linearity assuming a linear plant, comes from the discontinuous behavior of the relay function. In order to make the things more adequate and to meet the specifications more

\footnotetext{
${ }^{1}$ It is worth to mention that $G_{u}(s)$ can also be directly obtained from (5) as $G_{u}(s)=\frac{F(s)}{1+Z_{t h}(s) G_{l}(s)}$.
}

easily, this relay should be symmetric with output in $\{-1,1\}$. To use this symmetrical relay, the plant model should be modified to create a new control variable, $w$, that is binary also, and takes the values in $\{-1,1\}$. Accordingly, this controller will be implemented as an analog hysteretic comparator, with positive hysteresis of width $2 b$ and with values $\{-1,1\}$. So, the control loop will be modified as in Fig. 5.

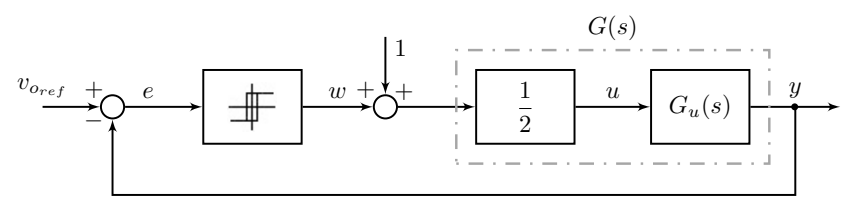

Fig. 5: Closed-loop control block diagram while $w$ is control signal.

Considering $u=(w+1) / 2$ mapping, the equations (6) and (10) will be converted to (13) and (14), respectively, as

$$
\begin{gathered}
C_{o} \dot{x}_{1}=-G_{R} x_{1}-\frac{P_{o}}{x_{1}}+x_{2}, \\
L \dot{x}_{2}=-x_{1}-r x_{2}+\frac{E}{2} w+\frac{E}{2}, \\
A=\left[\begin{array}{cc}
\frac{\bar{P}_{o}}{C_{o} \bar{v}_{o}^{2}}-\frac{G_{R}}{C_{o}} & \frac{1}{C_{o}} \\
-\frac{1}{L} & -\frac{r}{L}
\end{array}\right], B_{1}=\left[\begin{array}{c}
0 \\
\frac{E}{2 L}
\end{array}\right], B_{2}=\left[\begin{array}{c}
-\frac{1}{C_{o} \bar{v}_{o}} \\
0
\end{array}\right], \\
C=\left[\begin{array}{ll}
1 & 0
\end{array}\right], D=0,
\end{gathered}
$$

and the transfer function of the buck converter feeding mixed load will be $G(s)=\frac{Y(s)}{W(s)}=\frac{1}{2} G_{u}(s)$.

\section{A. Parallel Feed-forward Compensator}

The necessity of using PFC can be explained considering that we want to control a plant with relative degree 2 by using a relay controller. If we close the loop directly with the relay, we will obtain a closed-loop time response of the system that is not enough adequate to meet the specifications of the control strategy due to obtaining an output voltage with very high amplitude oscillations. So, we need to work with a more adequate plant with relative degree 1 and minimum phase characteristic to achieve the desired behavior. As our original plant does not meet these behavior we will rearrange this plant by using PFC. In addition, ASPR-ness condition of the plant can be guaranteed by adding a suitable parallel compensator $K(s)$ to the plant [7]. A linear plant $G(s)$ is almost strictly positive real (ASPR) [13], if and only if

1) Relative degree of $G(s)$ is $1, r d\{G(s)\}=1$,

2) $G(s)$ is minimum phase. It means that the zeros of $G(s)$ stay in the open LHP of the $s$-plane.

The desired parallel compensator $K(s)$ must satisfy the following conditions:

1) $K(s)$ must be stable,

2) Relative degree of $K(s)$ must be one, $\operatorname{rd}\{K(s)\}=1$.

In converter applications to achieve more adequate system, the output of the parallel compensator $K(s)$ should be considered negligible in steady-state with respect to the output of the linear plant $G(s)$. In addition, in this work, minimum order of $K(s)$ is more convenient to reduce the complexity of the 
augmented plant. An augmented plant $G_{a}(s)$, consisting of a linear plant with a parallel feed-forward compensator $K(s)$ is shown in Fig. 6.

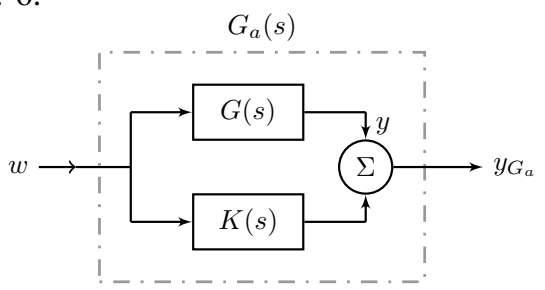

Fig. 6: Augmented plant, $G_{a}(s)$, the parallel connection of a linear plant, $G(s)$, with a compensator, $K(s)$.

In this case, we face a problem due to having a compensator in parallel with the plant, but fortunately this architecture is equivalent to another architecture as is illustrated in Fig. 7.

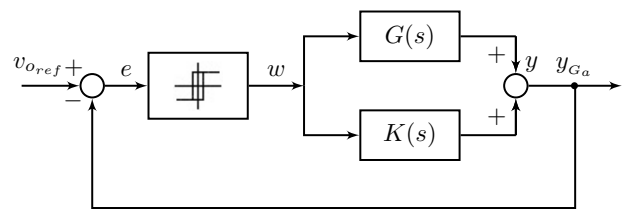

(a) Compensator $K(s)$ in forward path parallel with the plant

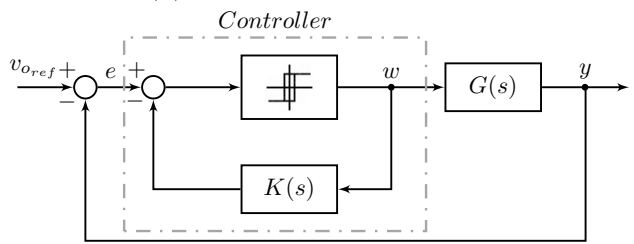

(b) Compensator $K(s)$ in feedback path with the controller

Fig. 7: Identical closed-loop block diagram.

Due to the block diagram rules, it is evident that the performance of a closed-loop system with the compensator in the forward path in parallel with the plant, Fig. $7 \mathrm{a}$, is identical with the compensator $K(s)$ in the feedback path of the controller, Fig. 7b. Taking into account the plant transfer function in (11), a suitable PFC for the CPL-buck converter is

$$
K(s)=\frac{k_{0} s}{s^{2}+c_{1} s+c_{0}} .
$$

Using the parameters $k_{0}, c_{1}$, and $c_{0}$, the zeros of the augmented plant can be placed at the desired positions in the open LHP for a range of $P_{o}$ values. For these purposes, by trial and error on the root locus diagram of the $G(s)$ in negative feedback with $K^{-1}(s), L(s)=K^{-1}(s) G(s)$, closedloop poles, which are the compensated-plant zeros, can be chosen to be stable and as damped as possible [7] and [16].

\section{B. LPRS-based Design of a Relay Controller}

In this work, the plant is linear, while the controller is a symmetrical positive hysteresis with output in $\{-c, c\}$ as is shown in Fig. 8

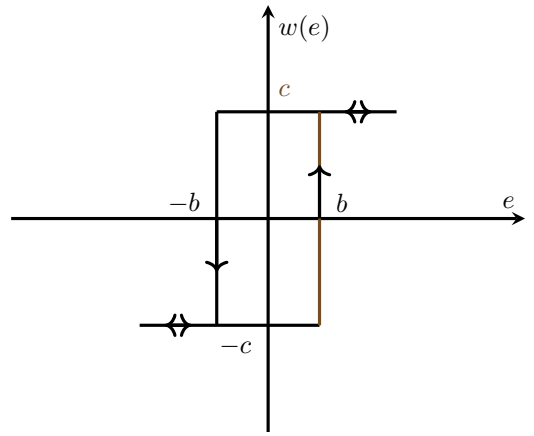

Fig. 8: Relay function that exhibits a hysteresis behavior.

$$
w=\left\{\begin{array}{l}
+c \text { if } e>-b, \quad w(t-\epsilon)=c, \\
-c \text { if } e<b, \quad w(t-\epsilon)=-c,
\end{array}\right.
$$

where $e$ is the error signal, $w=w(e)$ is the control signal, $2 b$ is the hysteresis width, and $c$ is the output amplitude of the relay and in particular, $c=1$, to represent the binary states of the switches. To evaluate the system stability, output oscillations and find the $b$ value of the relay, LPRS is used which is a frequency-domain analysis and provides the designer with exact information [7], and [14]. The locus of the perturbed relay system is the function $J(w)$ and it is defined as

$$
J(\omega)=-\frac{1}{2} \frac{1}{k_{e q}}+\left.j \frac{\pi}{4 c} y(t)\right|_{t=0},
$$

where $k_{e q}$ is the relay equivalent gain and is equal to $-1 / 2 \operatorname{Re}\{J(w)\}, c$ is the positive output value of the relay and $\left.y(t)\right|_{t=0}$ shows the relay switch condition in $t=0$ which is the time when the relay switch moves from $(-c)$ to $(+c)$. Once the system LPRS is computed, the possible limit cycle frequency and the corresponding equivalent gain of the relay element, $k_{e q}$, can be extracted by finding the $J(w)$ intersection with a horizontal line of ordinate $\pi b / 4 c$ and depending on $b$ value, lies below or above the horizontal axes, $(b>0)$ and $(b<0)$, respectively. After finding possible solution by LPRS, the stability of the periodic solution must be investigated. Derivation of LPRS relation is introduced in [14], and this relation is computed with Poincaré map technique which is a common way for computing periodic solution. The state-space expression of LPRS for a non-integrating and delay-free plant, as in the plant of this work [14], is

$$
\begin{aligned}
J(\omega)= & -0.5\left(A^{-1}+\frac{2 \pi}{\omega}\left(I-e^{(2 \pi / \omega) A}\right)^{-1} e^{(\pi / \omega) A}\right) B \\
& +j \frac{\pi}{4} C\left(I+e^{(\pi / \omega) A}\right)^{-1}\left(I-e^{(\pi / \omega) A}\right)^{-1} A^{-1} B .
\end{aligned}
$$

Note that (18) contains only the plant parameters and $\omega$ is the possible periodic solution frequency. For finding a solution in LPRS technique, at least one intersection point between the horizontal line and the $J(w)$ must exist. For this reason, at first by selecting relay parameter $b$, the start point of horizontal line in $j \omega$ axis is found, then extend it to $-\infty$ in horizontal axes till an intersection occurs. If there is no intersection for this try, other relay parameter $b$ is selected and this procedure will be repeated. In this article, instead of mentioned procedure, the 
relay parameter $b$ is found by moving the horizontal line over $j \omega$ axis and find all possible intersection points. Then select an intersection point with minimum $\operatorname{Re}\{J(\omega)\}$ as a solution. So, the relay parameter $b$ is

$$
b=-\frac{4 c}{\pi} \operatorname{Im}\{J(\omega)\} .
$$

In a relay control system, without considering the autooscillations of the loop, the dynamics of the system can be divided into two sub-systems [7] and [14]. One is the fast sub-system that refers to the behavior of the loop with the relay and the other one is the slow sub-system that refers to the propagations of the reference and disturbance signals through the loop. In the control loop with symmetrical relay, in Fig.5, there will be an input that behaves as a disturbance in the control signal. This disturbance affects the behavior of the system and will create a steady-state error with respect to the system reference, so the probable difference between the mean value of the output with respect to the reference comes from this disturbance. As this effect appears in the slow subsystem dynamics, linear slow motions of the system should be considered. For this purpose, the relay element should be substituted by $k_{e q}$, which is the relay equivalent gain. The block diagram for the system taking into account only the slow motions is shown in Fig. 9.

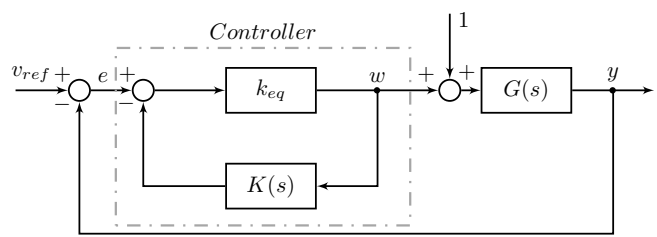

Fig. 9: Equivalent block diagram for the system slow sub-system.

It is noticeable that this steady-state error is small when the equivalent gain, $k_{e q}$, is large.

\section{Controller Design}

This section presents the design of the controller from the linearized model of the plant. The parameters of the buck converter with mixed load are: $E=48 \mathrm{~V}, r=0.05 \Omega$, $L=100 \mu \mathrm{H}$, and $C_{o}=470 \mu \mathrm{F}$. The desired objective of the controller is an output voltage equal to, $\bar{v}_{o}=24 \mathrm{~V}$. With respect to (10), the values of the state-space matrices for the linearized system A, B, C, and D for $G_{R}=0.01 \mho, P_{o}=135$ $\mathrm{W}$, and with $\Delta P_{o}=0$ are

$$
\begin{gathered}
A=\left[\begin{array}{cc}
477.3936 & 2.1277 \cdot 10^{3} \\
-10000 & -500
\end{array}\right], B_{1}=\left[\begin{array}{c}
0 \\
24 \cdot 10^{4}
\end{array}\right], \\
C=\left[\begin{array}{ll}
1 & 0
\end{array}\right], D=0 .
\end{gathered}
$$

The curves of the poles values when $P_{o} \in[0,3000] \mathrm{W}$ are shown in Fig. 10, with Fig. 10a showing the real parts of the plant open-loop poles and Fig. 10b their imaginary parts. Fig. 10a shows that the plant has only negative real parts when the output power is lower than $135 \mathrm{~W}$ which is a very low value. So, for enlarging the output power of the converter, a suitable closed-loop controller should be designed.

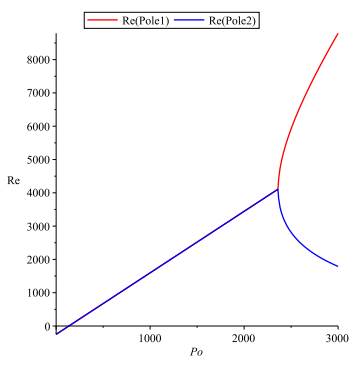

(a) $\operatorname{Re}\{$ poles $\}$

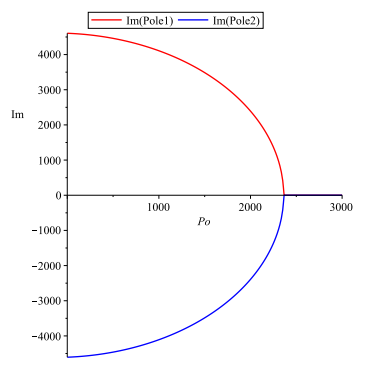

(b) $\operatorname{Im}\{$ poles $\}$
Fig. 10: Augmented poles with respect to $P_{o} \in[0,3000] \mathrm{W}$.

The maximum possible open-loop output power of the plant, $P_{o_{\max }}$, is about $11500 \mathrm{~W}$. As mentioned in section III, some suitable PFCs fulfill the plant relative degree requirement, make compensated-plant minimum-phase and improve the stability of the closed-loop system regarding to the output power. So, with trial and error on the root locus of the openloop transfer function, $L(s)=K^{-1}(s) G(s)$, in $P_{o}=200 \mathrm{~W}$, a suitable PFC is designed as

$$
K(s)=\frac{3.7547 \cdot 10^{4} s}{s^{2}+6312.0 s+1.856 \cdot 10^{7}} .
$$

The root locus of the open-loop transfer function of the plant, $L(s)$ is shown in Fig. 11. In this figure, the closed-loop poles, that will be the zeros of the augmented plant, $G_{a}(s)=$ $K(s)+G(s)$, are marked with squares and they correspond to an open-loop gain value of $2.6633 \cdot 10^{-5}$, which finally appears in the compensator $K(s)$. In the simulation results of the next section, it is shown that how the designed PFC in (20) improves the upper limit in (12).

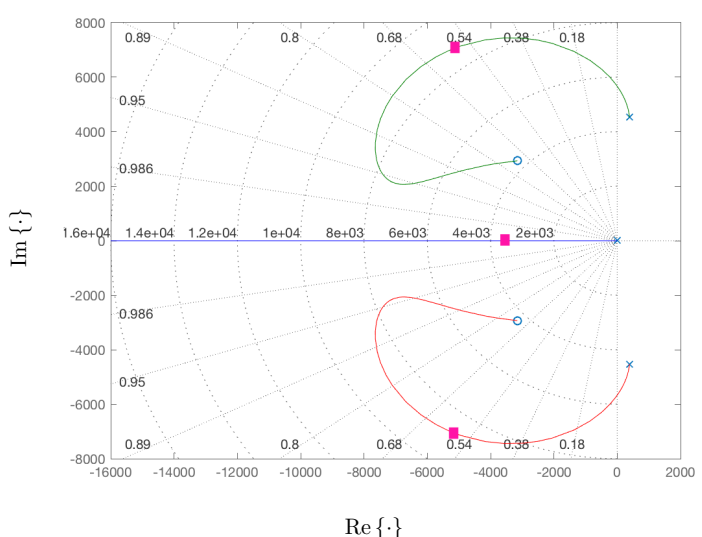

Fig. 11: Root locus of $L(s)=K^{-1}(s) G(s)$.

To design the relay parameters $b$ and $c$, the LPRS technique is used. In converter applications, parameter $c$ is one, while parameter $b$ must be designed to fulfill the desired specifications. As mentioned before, the plant is stable for $P_{o} \leq 135 \mathrm{~W}$ but to evaluate the LPRS behavior regarding to $P_{o}$, Fig. 12 is used. The $J(w)$ curves for $\omega \in[6283, \infty) \mathrm{rad} / \mathrm{s}$ for power values $P_{o}=20 \mathrm{~W}$ (stable plant), $P_{o}=135 \mathrm{~W}$ (marginally stable plant), and $P_{o}=1500 \mathrm{~W}$ (unstable plant) of the augmented 
plant are shown in Fig. 12. Considering this figure, the LPRS curve in stable mode stays under the horizontal axis, while in unstable mode, for lower frequencies, it is above of the horizontal axis.

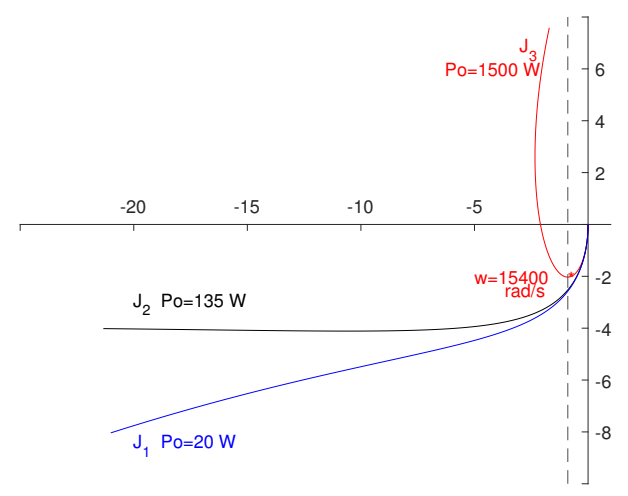

Fig. 12: $J(w)$ curves for stable, $J_{1}$, marginally stable, $J_{2}$, and unstable, $J_{3}$, plants.

In LPRS technique, if there is a solution, (intersection point), the parameter $b$ is obtained from the intersection of the horizontal line and LPRS curve, $J(\omega)$. In this article, for selecting relay parameter $b$, a new approach is introduced. In this approach by moving the horizontal line on the $j \omega$ axis, some intersection points may be produced. The value of the relay parameter $b$ is selected from these intersection points where the selected intersection point will be the one with minimum real part. In this manner, the equivalent gain of the relay will be maximum and then, the dynamics of the global closed-loop system will be very close to the dynamics associated to the zeros of $G_{a}(s)$. In addition, as Fig. 12 shows, for any output power in stable or unstable mode for $\omega \in[15400, \infty) \mathrm{rad} / \mathrm{s}$, by increasing $\omega$, the real part of LPRS, $\operatorname{Re}\{J(\omega)\}$, will decrease. Accordingly, the designer should select a solution in this region with switching frequency up to its maximum allowable value. For selecting the relay parameter $b$, the LPRS curve of the $G_{a}(s)$, is considered for $\omega \in[1257, \infty) \mathrm{rad} / \mathrm{s}$ with $P_{o}=200 \mathrm{~W}$. In Fig. 13, the LPRS curve of the augmented plant with horizontal line is shown.

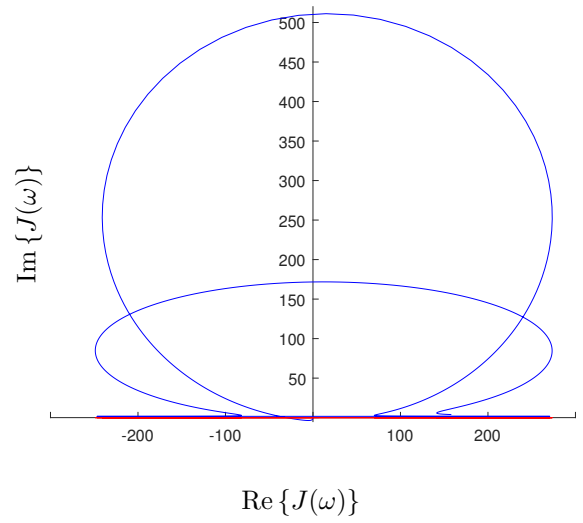

Fig. 13: $J(w)$ for the augmented plant, $G_{a}(s)$, and $P_{o}=200 \mathrm{~W}$.
The zoom plot of Fig. 13 in the intersection point where the relay parameter is $b=0.0760$ and $k_{e q}=5.2174 \cdot 10^{3}$ is shown in Fig. 14.

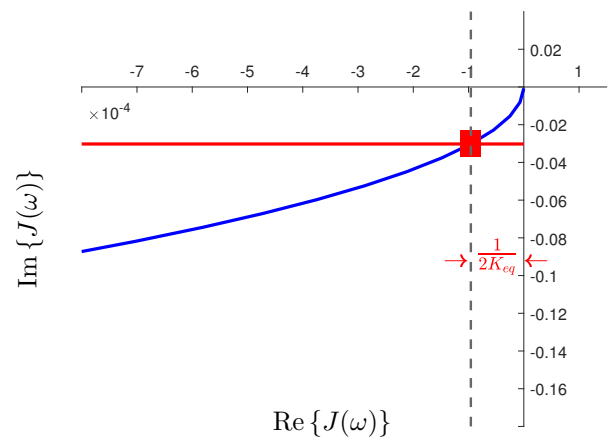

Fig. 14: Zoom of Fig. 13 in the intersection point.

The solution gives $\omega=775700 \mathrm{rad} / \mathrm{s}$ or equivalent frequency $f=123.46 \mathrm{kHz}^{2}$. Table I shows the comparison of the output voltage ripples and switching frequencies with LPRS solution technique and the non-linear model simulation results of the following section. As it was expected [14], the LPRS technique results are approximately very close to the practical results even with the non-linear CPL model.

TABLE I: Fast sub-system frequency and ripple.

\begin{tabular}{ccc}
\hline & Ripple $(\mathrm{V})$ & $f(\mathrm{kHz})$ \\
\hline LPRS solution & - & 123.46 \\
Non-linear CPL model & $1.5 \cdot 10^{-3}$ & 120.48 \\
\hline
\end{tabular}

\section{Simulation Results}

This section presents some numerical simulations that illustrate the behavior of the closed-loop system under different load values, $P_{o}$, and input voltages, $E$. In the simulations ${ }^{3}$, the closed-loop non-linear model of the plant is used. The output voltage of the system, $v_{o}$, for the parameters that are stated in previous section with $P_{o}=100 \mathrm{~W}$ is shown in Fig. 15 while $v_{o_{\text {ref }}}$ is the output voltage reference which is $24 \mathrm{~V}$ and $v_{o_{a v g}}$ is the average of the system output voltage ripple ${ }^{4}$. As it is clear from this figure, the start up of the system is quite good and without critical oscillations.

\footnotetext{
${ }^{2}$ It is worth to mention that, for the plant parameters, the natural frequency of the converter $L C$ filter is around $f_{n}=739 \mathrm{~Hz}$

${ }^{3}$ In this work, Simulink/Matlab has been used to do all the numerical simulations.

${ }^{4}$ In the figures that include the output voltage in this section, for more clearness, the control signal $u$ is not shown.
} 


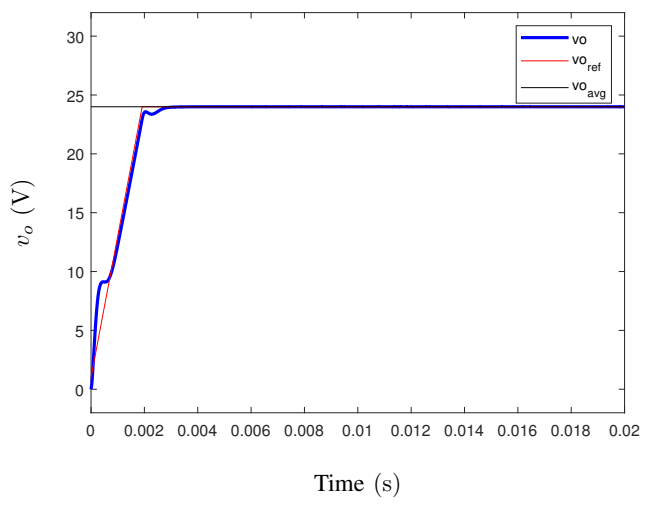

Fig. 15: $v_{o}$ of the closed-loop system.

To make it easier to see the ripple amplitude and the frequency of the output voltage, the zoom plot of the steadystate region, with the scaled control signal $u$, is shown in Fig. 16. This figure shows that the maximum amplitude of the ripple is $2 \mathrm{mV}$ and the average of the output voltage ripple is very close to the output voltage reference even with the non-linear model.

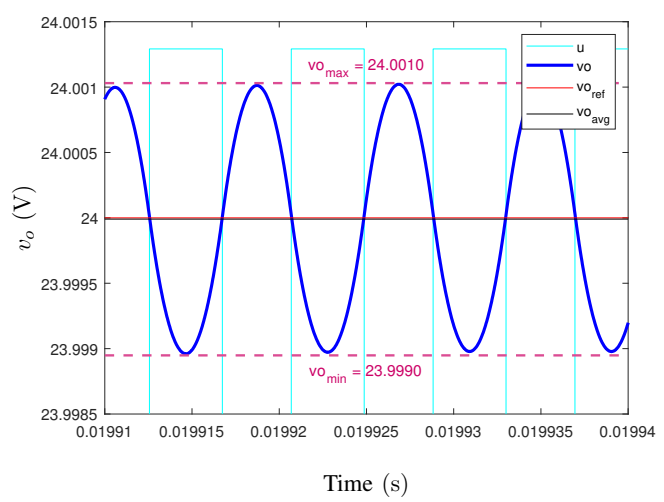

Fig. 16: Zoom plot of the steady-state region in Fig. 15.

To go one step further and to investigate the effect of uncertainties and disturbances on this system, the output voltage of the closed-loop system with variations in the converter input voltage, $E$, is shown in Fig. 17.

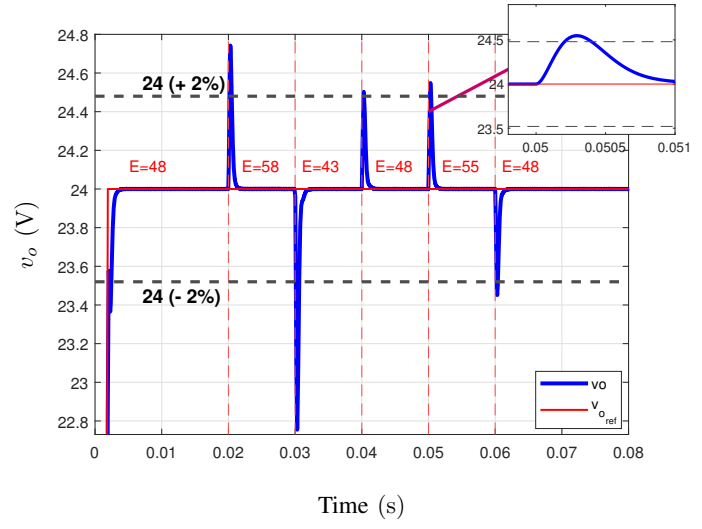

Fig. 17: $v_{o}$ of the closed-loop system with changes in $E$.
This figure shows the output voltage, $v_{o}$, due to the input voltage, $E$, variations, changes its value in a step manner. Besides, Fig. 17 shows the zoom plot of one of the variations, when $E$ changes from $48 \mathrm{~V}$ to $55 \mathrm{~V}$. This zoom plot shows that the maximum deviation in this change is around $0.55 \mathrm{~V}$ and it takes only $1 \mathrm{~ms}$ to attenuate this disturbance and reach the desired steady-state again.

The response of the closed-loop system with respect to the load power, $P_{o}$, changes is shown in Fig. 18. In this figure, for more clearness, the zoom plot of one of the output voltage variation while $P_{o}$ changes from $420 \mathrm{~W}$ to $810 \mathrm{~W}$ is shown. This zoom plot shows that the maximum voltage deviation in this change of the CPL power is approximately $1.8 \mathrm{~V}$ and it takes only $1 \mathrm{~ms}$ to reach again its desired value.

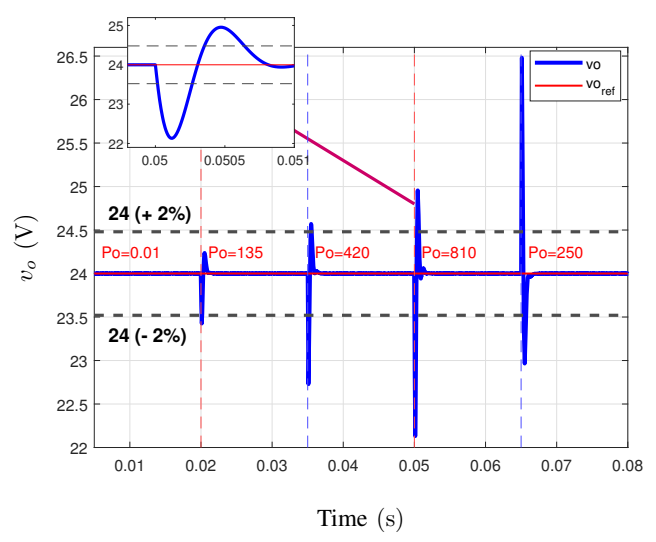

Fig. 18: $v_{o}$ of the closed-loop system with different loads $\left(P_{o}\right)$.

At a last test, disturbances in $E$ and $P_{o}$ are applied simultaneously to the system. The response of the closed-loop system while $E$ and $P_{o}$ change is shown in Fig. 19.

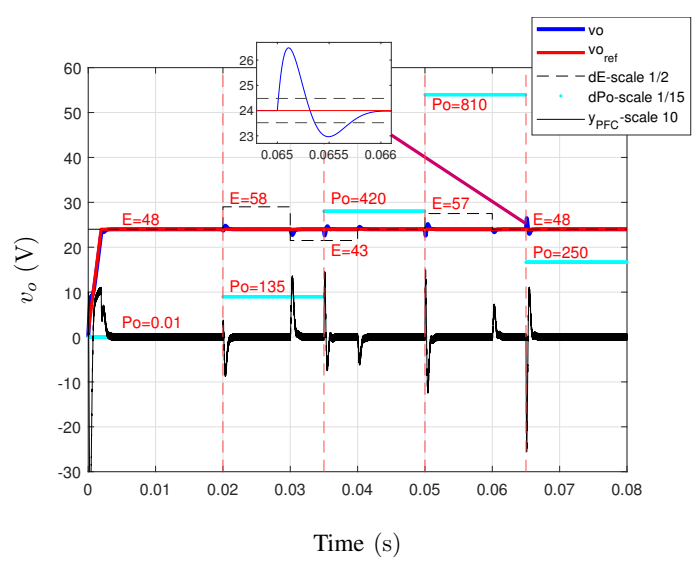

Fig. 19: $v_{o}$ of the closed-loop system when $P_{o}$ and $E$ are changing.

In Fig. 19, $y_{P F C}$, which is the output of the designed PFC, is scaled 10 times. This figure shows that $y_{P F C}$ is negligible in steady-state with respect to the plant output; moreover, the output of the compensator is different from zero only when the system is in a transient and in steady-state is equal to zero 
due to the existence of a pure derivative in the compensator $K(s)$, so it works as we desired. The zoom plot in this figure shows the voltage deviation when $E$ changes from $57 \mathrm{~V}$ to Time $\in[0,0.019] \mathrm{s}$ and $P_{o}$ changes from $810 \mathrm{~W}$ to $250 \mathrm{~W}$. It is clear that the most important change in output voltage occurs at time $0.065 \mathrm{~s}$ where $P_{o}$ has its biggest variation.

As a final simulation result, the effect of a band-limited white noise in the feedback path of the closed-loop system appears in Fig. 20. This figure shows the behavior of the output voltage before and after applying the noise, with more than $4 \mathrm{~V}$ amplitude, at time $0.02 \mathrm{~s}$. The zoom plot in Fig. 20, shows the output voltage response for Time $\in[0.015,0.025] \mathrm{s}$ with and without the noise. The result shows the good ability of the closed-loop system in attenuating the measurement noise.

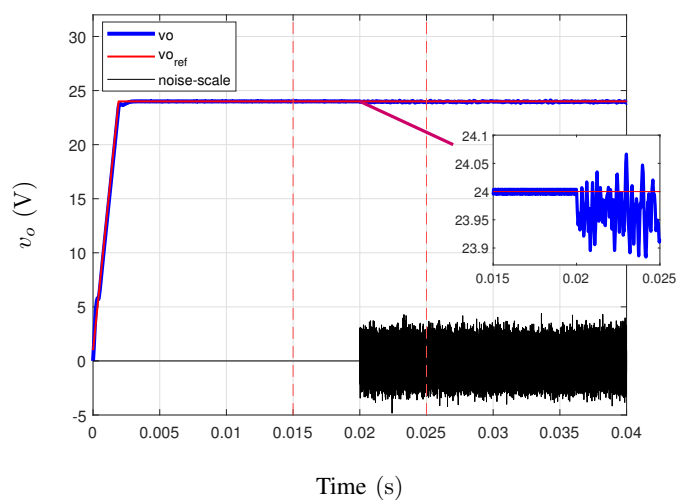

Fig. 20: $v_{o}$ of the closed-loop system with and without measurement noise.

\section{CONCLUSION}

Design procedure and simulation results in this work show that using PFC and LPRS is a suitable technique for designing relay controllers. The use of PFC allows to generate an adequate augmented plant for the outer relay control loop. In the studied case, this kind of control allows to enlarge the range of output power with stable operation of the buck converter in front of the open-loop case. Finally, the simulation results with the non-linear model of the plant show that the closed-loop system has a good performance even with input voltage and output power disturbances.

\section{ACKNOWLEDGMENT}

This work has been partially supported by the Government of Spain through the Agencia Estatal de Investigación Projects DPI2017-85404-P and DPI2017-89153-P (AEI/FEDER, UE), and by the Generalitat de Catalunya through the Project 2017 SGR 872.

\section{REFERENCES}

[1] Rahimi, A.M., Williamson, G.A. and Emadi, A., 2010. Loopcancellation technique: A novel nonlinear feedback to overcome the destabilizing effect of constant-power loads. IEEE Transactions on vehicular technology, 59(2), pp.650-661.
[2] Kwasinski, A. and Onwuchekwa, C.N., 2011. Dynamic behavior and stabilization of DC microgrids with instantaneous constant-power loads. IEEE Transactions on power electronics, 26(3), pp.822-834.

[3] Zhao, Y., Qiao, W. and Ha, D., 2014. A sliding-mode duty-ratio controller for DC/DC buck converters with constant power loads. IEEE Transactions on industry Applications, 50(2), pp.1448-1458.

[4] Su, M., Liu, Z., Sun, Y., Han, H. and Hou, X., 2018. Stability analysis and stabilization methods of DC microgrid with multiple parallelconnected DCDC converters loaded by CPLs. IEEE Transactions on Smart Grid, 9(1), pp.132-142.

[5] Verma, S., Singh, S. and Rao, A., 2013. Overview of control Techniques for DC-DC converters. Research Journal of Engineering Sciences ISSN, 2278, p. 9472.

[6] Boiko, I., 2016. LPRS analysis of sliding mode buck converter. Journal of the Franklin Institute, 353(18), pp.5137-5150.

[7] Cóbreces, S. and Griñó, R., 2016. Hysteretic control of grid-side current for a single-phase LCL grid-connected voltage source converter. Mathematics and Computers in Simulation, 130, pp.194-211.

[8] Deng, M., Iwai, Z. and Mizumoto, I., 1999. Robust parallel compensator design for output feedback stabilization of plants with structured uncertainty. Systems and control letters, 36(3), pp.193-198.

[9] Iwai, Z., Mizumoto, I. and Deng, M., 1994, December. A parallel feedforward compensator virtually realizing almost strictly positive real plant. In Proceedings of 1994 33rd IEEE Conference on Decision and Control (Vol. 3, pp. 2827-2832). IEEE.

[10] Mizumoto, I., Takagi, T. and Yamanaka, K., 2013, June. Parallel feedforward compensator design and ASPR based adaptive output feedback control for a time-delay system. In 2013 American Control Conference (pp. 4909-4914). IEEE.

[11] Ohtsuka, H., Nagata, M. and Iwai, Z., 2004, July. Sliding mode control system design using parallel feedforward compensator. In 2004 5th Asian Control Conference (IEEE Cat. No. 04EX904) (Vol. 3, pp. 19641972). IEEE.

[12] Kim, H., Kim, S., Back, J., Shim, H. and Seo, J.H., 2016. Design of stable parallel feedforward compensator and its application to synchronization problem. Automatica, 64, pp.208-216.

[13] Rusnak, I. and Barkana, I., 2009. SPR and ASPR untangled. IFAC Proceedings Volumes, 42(6), pp.126-131.

[14] Boiko, I., 2008. Discontinuous control systems: frequency-domain analysis and design. Springer Science \& Business Media.

[15] TSypkin, I.Z. and Tsypkin, Y.Z., 1984. Relay control systems. CUP Archive.

[16] Rusnak, I. and Barkana, I., 2012, November. The duality of parallel feedforward and negative feedback. In 2012 IEEE 27th Convention of Electrical and Electronics Engineers in Israel (pp. 1-4). IEEE. 